\title{
Density-functional study of tilt-angle and temperature-dependent conductance in biphenyl dithiol single-molecule junctions
}

\author{
F. Pauly, ${ }^{1,2, *}$ J. K. Viljas, ${ }^{1,2}$ J. C. Cuevas, ${ }^{1,2,3}$ and Gerd Schön ${ }^{1,2}$ \\ ${ }_{1}^{1}$ Institut für Theoretische Festkörperphysik and DFG-Center for Functional Nanostructures, Universität Karlsruhe, \\ 76128 Karlsruhe, Germany \\ ${ }^{2}$ Institut für Nanotechnologie, Forschungszentrum Karlsruhe, 76021 Karlsruhe, Germany \\ ${ }^{3}$ Departamento de Física Teórica de la Materia Condensada, Universidad Autónoma de Madrid, 28049 Madrid, Spain
}

(Received 22 May 2007; revised manuscript received 11 February 2008; published 9 April 2008)

\begin{abstract}
In the framework of a density-functional-based method, we study electron transport through several biphenyl-derived dithiol molecules connected to gold electrodes. The molecules differ in the degree of conjugation of the $\pi$-electron system, which is controlled by the choice of the side groups. The low-temperature

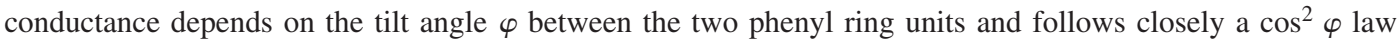
consistent with an effective $\pi$-orbital coupling model. Tilting the phenyl rings from a planar conformation to one with perpendicular rings decreases the conductance by roughly 2 orders of magnitude. These findings are in agreement with experiments. We further study the temperature dependence of both the conductance and its fluctuations and find qualitative differences between the investigated molecules. The temperature dependence arises from thermal smearing in the leads and also from a thermal average over different contact configurations. Our results suggest that the variations of the conductance due to the latter effect can be reduced by an appropriate design of the molecule.
\end{abstract}

DOI: $10.1103 /$ PhysRevB.77.155312

PACS number(s): 73.63.Rt, 73.23.Ad, 73.40.-c

\section{INTRODUCTION}

The electron transport through molecular junctions depends on the precise arrangement of the atoms in the system, and many of the molecule-specific applications and potential advantages over other nanoelectronic systems rely on the property that molecules with tailored atomic configuration can be synthesized reproducibly. ${ }^{1}$ However, in many cases, the conductance is similarly sensitive to the details of the contact of the molecule to the electrodes. ${ }^{2-7}$ They are usually not well controlled, which complicates the reproducibility of the experiments. ${ }^{8-12}$ For molecules which are not rigid, further variations and uncertainties arise. ${ }^{12-16}$ This effect strongly depends on temperature. ${ }^{17,18}$ Information obtained from single-molecule transport experiments is therefore mostly of a statistical nature, which is represented in conductance histograms..$^{3,9,11,19-21}$ For these reasons, it is important to study families of molecules, the internal structure of which can be systematically varied but can still couple similarly to the electrodes. With this motivation, in the present work, we analyze the charge transport through various biphenyl-derived dithiol (BPDDT) molecules with different molecular conformations, which are connected to gold electrodes in the same way.

Recently, Venkataraman et al. ${ }^{22}$ measured the influence of molecular conformation on the conductance of biphenylderived molecules, where different side groups were used to control the tilt angle $\varphi$ between two phenyl rings. In this way, the extent of the delocalized $\pi$-electron system of the molecules could be varied. They found that the conductance exhibited a characteristic $\cos ^{2} \varphi$ dependence, as expected from a simple effective $\pi$-orbital coupling model. ${ }^{23,24}$ Their observations were supported by a theoretical estimate of the effect based on the splitting of frontier orbitals. ${ }^{22}$

In this work, we theoretically analyze the charge-transport properties of three different BPDDT molecules connected to gold electrodes. We refer to the molecules as R2, S2, and D2 (Fig. 1). While R2 is the conventional biphenyl, the other two molecules, 2,2' -dimethyl-biphenyl (S2) and 2,6,2',6'tetramethyl-biphenyl (D2), incorporate one or two methyl groups in the ortho position with respect to the ringconnecting carbon atoms. We describe the electronic structure of the single-molecule contacts at the level of densityfunctional theory (DFT) and, by using Green's function techniques, we compute the conductance within the Landauer-Büttiker formalism. We determine the dependence of the conductance on both the tilt angle and the temperature.

By varying the degree of $\pi$ conjugation by changing the

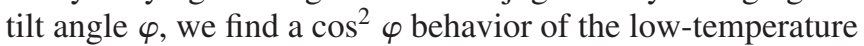
conductance, which is in agreement with the experiments of Ref. 22. This behavior is, by and large, independent of the methyl side groups attached. Breaking the conjugation reduces the low-temperature conductance by roughly 2 orders of magnitude. At higher temperatures, two contributions to the temperature dependence of the conductance are important. The first one comes from the thermal broadening of the lead properties, while the other one comes from a thermal average over different geometric configurations of the contacts. We observe that the conductances of S2 and D2 monotonously grow with increasing temperature, while for R2,

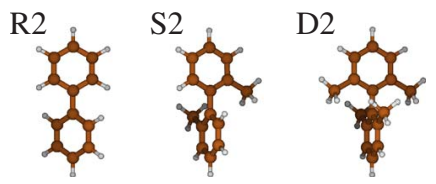

FIG. 1. (Color online) Biphenyl molecules R2, S2, and D2. For $\mathrm{S} 2$, one hydrogen atom in the ortho position with respect to the ring-connecting carbons in each phenyl ring of $\mathrm{R} 2$ is replaced by a methyl group. For D2, also the second ortho-positioned hydrogen is substituted by a methyl group. 
the temperature dependence is weak and nonmonotonous. We demonstrate how the temperature dependence of the conductance and its fluctuations can be modified by an appropriate design of the geometric structure of a molecule.

Motivated by the synthesis of the BPDDT molecules R2 and S2 reported in Ref. 25, which are now available for transport measurements, here we investigate molecules coupled to gold electrodes via thiol end groups. We note that different end groups may modify the absolute values of the conductance; ${ }^{9-11}$ however, the tilt-angle dependence is primarily determined by the internal structure of the molecule. Therefore, we also compare our results to the data presented in Ref. 22, although in that case amino groups were used for the coupling.

The paper is organized as follows. In Sec. II, we outline the methods used to compute the electronic structure, the geometry, and the conductance of the molecular contacts discussed below. In Sec. III we discuss the conductance of the three BPDDT molecules, particularly its tilt-angle and temperature dependence. Some technical details are deferred to the Appendix. Finally, in Sec. IV, we summarize our results.

\section{THEORETICAL MODEL}

In this section, we present the methods for computing the electronic structure, the contact geometries, and the transport properties of the molecular contacts. For further details on our method, we refer the reader to Refs. 26-28.

\section{A. Electronic structure and contact geometries}

We determine the electronic structure from DFT as implemented in the RI-DFT module of the quantum chemistry package TURBOMOLE V5.7. ${ }^{29,30}$ All calculations, including the description of the electrodes, are performed within the TURBOMOLE standard Gaussian basis set, which is of splitvalence quality with polarization functions on all nonhydrogen atoms. ${ }^{30-32}$ As an exchange-correlation functional we use BP86. ${ }^{33-35}$ All calculations are performed in a closed-shell formalism, and total energies are converged to a precision better than $10^{-6}$ a.u.

The contact geometries are displayed in Fig. 2. They are determined by first calculating the geometric structure of a gold $(\mathrm{Au})$ [111] pyramid with a thiolated benzene molecule on top [Fig. 3(a)]. This pyramid consists of three layers of Au with three, six, and ten atoms. The tip atom of the pyramid is missing so that the sulfur (S) atom of the benzene binds to a threefold hollow site on top of the Au structure. We relax all atomic positions except for the layers containing six and ten atoms, which are kept fixed with the lattice constant set to the bulk value of $4.08 \AA$, which is obtained from experiment. Next, we compute the geometry of the biphenyl molecules R2, S2, and D2 of Fig. 1 with the hydrogen atoms in the position 4 and $4^{\prime}$ substituted by $\mathrm{S}$ atoms, which are bonded to a single Au atom, respectively [Fig. 3(b)]. For each of these molecules, we replace the single Au atom on each side with the mentioned Au [111] pyramids, where the thiolated benzene molecule has been removed [Fig. 3(c)]. In this process, we take care that the $\mathrm{S}$ atoms of the biphenyl
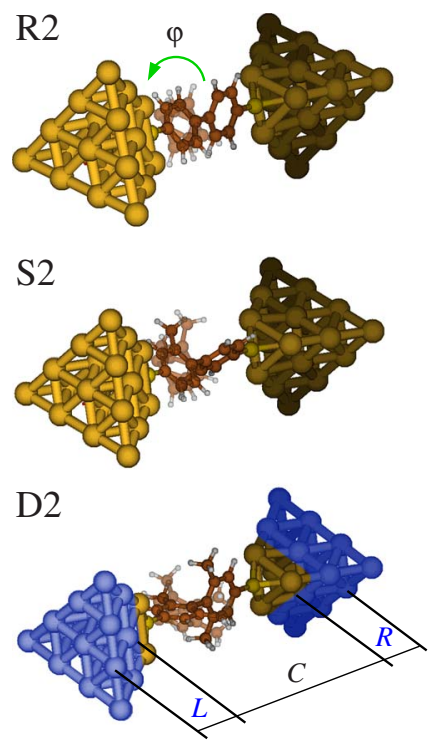

FIG. 2. (Color online) Molecules R2, S2, and D2 contacted at both ends to $\mathrm{Au}$ [111] electrodes via a sulfur atom in a threefold binding position. Faintly overlaid on the ground-state structure of the single-molecule contacts are geometries, where the left ring is rotated to $\varphi=\varphi_{0} \pm 30^{\circ}$. Here, $\varphi$, as indicated for R2, is the tilt angle between the planes of the two phenyl rings and $\varphi_{0}$ is its groundstate value. The division of the junctions into the left $(L)$, central $(C)$, and right $(R)$ regions, which is used in the conductance calculations, is also shown.

molecules are in the old positions of the $S$ atoms of the thiolated benzene on top of the Au pyramids and that the S-S molecular axis and the [111] direction are aligned. The ground-state geometry is subsequently obtained by relaxing the complete structure, where we fix only the two terminal gold layers on each side [Fig. 3(c)]. As mentioned above, the lattice constant in these layers is $4.08 \AA$. In the process, we let the maximum norm of the Cartesian gradient decay to values below $10^{-4}$ a.u.

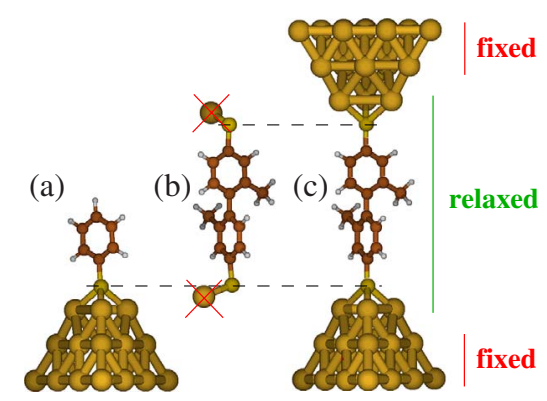

FIG. 3. (Color online) Construction of the contact geometry for molecule S2. (a) Geometric structure of a thiolated benzene molecule on top of a Au pyramid, (b) molecule S2 with Au-S termination, and (c) contact geometry for molecule S2. The geometries correspond to ground-state structures except for the Au [111] pyramids. As indicated, for them, the inner three atoms are relaxed, whereas the outer two layers are kept fixed. The S-S distance of geometry (b) is roughly the same as in the contact geometry (c) (see the dashed lines). 


\section{B. Transmission function}

We compute the charge transport by using the Landauer formula and Green's functions expressed in a local nonorthogonal basis. ${ }^{27,36,37}$ The local basis allows us to partition the basis states into left $(L)$, central $(C)$, and right $(R)$ parts, according to a division of the contact geometry. Thus, the Hamiltonian (or Kohn-Sham) matrix $\boldsymbol{H}$ and analogously the overlap matrix $S$, can be written in block form,

$$
\boldsymbol{H}=\left(\begin{array}{ccc}
\boldsymbol{H}_{L L} & \boldsymbol{H}_{L C} & \mathbf{0} \\
\boldsymbol{H}_{C L} & \boldsymbol{H}_{C C} & \boldsymbol{H}_{C R} \\
\mathbf{0} & \boldsymbol{H}_{R C} & \boldsymbol{H}_{R R}
\end{array}\right) .
$$

Within the Green's function method, the energy-dependent transmission $\tau(E)$ is expressed as ${ }^{38}$

$$
\tau(E)=\operatorname{Tr}\left[\boldsymbol{\Gamma}_{L} \boldsymbol{G}_{C C}^{r} \boldsymbol{\Gamma}_{R} \boldsymbol{G}_{C C}^{a}\right],
$$

with the Green's functions

$$
\boldsymbol{G}_{C C}^{r}(E)=\left[E \boldsymbol{S}_{C C}-\boldsymbol{H}_{C C}-\boldsymbol{\Sigma}_{L}^{r}(E)-\boldsymbol{\Sigma}_{R}^{r}(E)\right]^{-1}
$$

and $\boldsymbol{G}_{C C}^{a}=\left[\boldsymbol{G}_{C C}^{r}\right]^{\dagger}$, the self-energies

$$
\boldsymbol{\Sigma}_{X}^{r}(E)=\left(\boldsymbol{H}_{C X}-E \boldsymbol{S}_{C X}\right) \boldsymbol{g}_{X X}^{r}(E)\left(\boldsymbol{H}_{X C}-E \boldsymbol{S}_{X C}\right),
$$

the scattering rate matrices $\boldsymbol{\Gamma}_{X}(E)=-2 \operatorname{Im}\left[\boldsymbol{\Sigma}_{X}^{r}(E)\right]$, and the electrode Green's function $\boldsymbol{g}_{X X}^{r}$, with $X=L$ and $R$.

In Fig. 2, we show how we divide our contacts into the $L$, $C$, and $R$ regions. In this way we obtain $\boldsymbol{H}_{C C}$ and $\boldsymbol{S}_{C C}$ for the $C$ region, which consists of the BPDDT molecule and three gold atoms on each side of the junction. The $L$ and $R$ regions are semi-infinite electrodes, which we model through Green's functions $\boldsymbol{g}_{X X}^{r}(E)$ calculated for surfaces of ideal semi-infinite crystals. The two terminal gold layers on each side of the gold pyramids ( $L$ and $R$ regions in Fig. 2 ) are the parts of the surfaces that are assumed to couple to $C$. The matrices $\boldsymbol{H}_{C X}$ and $\boldsymbol{S}_{C X}$ are extracted from the finite contact geometries.

In order to obtain $\boldsymbol{g}_{X X}^{r}(E)$, we separately compute the electronic structure of a spherical gold cluster with 429 atoms (not shown). From this cluster, we extract "bulk parameters" and construct a semi-infinite crystal that is infinitely extended perpendicular to the transport direction. ${ }^{26}$ The surface Green's functions are then calculated from this crystal using the so-called decimation technique. ${ }^{39}$ We have checked that the electrode construction converged with respect to the size of the Au cluster, from which we extract our parameters. ${ }^{26}$ In this way we consistently describe the whole system within DFT by using the same nonorthogonal basis set and exchange-correlation functional everywhere.

We assume the Fermi energy $E_{F}$ to be fixed by the gold leads, for which we obtain $E_{F}=-5.0 \mathrm{eV}$ from the spherical $\mathrm{Au}_{429}$ cluster. The proper alignment of the molecular orbitals in the $C$ region ${ }^{40-42}$ is accounted for by computing the larger contact geometries shown in Fig. 2.

\section{Conductance}

The linear conductance in the Landauer formalism is given by ${ }^{15,38}$

$$
G_{\varphi}(T)=G_{0} \int_{-\infty}^{\infty} d E\left[-\partial_{E} f(E, T)\right] \tau_{\varphi}(E),
$$

where $G_{0}=2 e^{2} / h$ is the conductance quantum and $\tau_{\varphi}(E)$ is the transmission [Eq. (2)]. ${ }^{43}$ Here and henceforth, $G$ and $\tau$ carry the index $\varphi$, which parametrizes the tilt angle $\varphi$ between two phenyl rings (Fig. 2). For zero temperature, Eq. (5) reduces to $G_{\varphi}(T=0 \mathrm{~K})=G_{0} \tau_{\varphi}\left(E_{F}\right)$. In general, we average over an energy window of the order of $k_{B} T$, as described by the derivative of the Fermi function, $-\partial_{E} f$. This averaging accounts for the "electronic" or "lead-induced" thermal broadening. ${ }^{38}$ It is to be distinguished from a second temperature-dependent effect, which arises from deviations of the tilt angles $\varphi$ from the minimum-energy ground-state value. These fluctuations further modify the conductance ${ }^{14,15}$

$$
\bar{G}(T)=\left\langle G_{\varphi}(T)\right\rangle_{\varphi}
$$

with $\langle\cdots\rangle_{\varphi}=\int d \varphi e^{-E_{\varphi} / k_{B} T}(\cdots) / \int d \varphi e^{-E_{\varphi} / k_{B} T}$. In this expression, $E_{\varphi}$ is the electronic ground-state energy of the metalmolecule-metal contact for angle $\varphi$. The $\varphi$ average leads to a "configurational" temperature dependence of $\bar{G}(T)$. For later use, we also introduce the variance, which describes the fluctuations of the conductance as follows:

$$
\delta G(T)=\sqrt{\left\langle\left(G_{\varphi}(T)-\bar{G}(T)\right)^{2}\right\rangle_{\varphi}} .
$$

\section{RESULTS}

In this section, we present the results of our DFT-based transport approach. We discuss the properties of the isolated molecules, the conductance of Au-molecule-Au contacts in the ground-state configuration, and the tilt-angle dependence of both the ground-state energy and the low-temperature conductance. Then, we address the central issue of this work, namely, the temperature dependence of the conductance and its fluctuations. The latter results are explained by using an analytical expansion of the conductance valid at low temperatures.

\section{A. Isolated molecules}

First, we discuss some properties of the isolated molecules (Fig. 1). For R2, S2, and D2, we obtain the phenyl ring tilt angles $\varphi$ of $36.4^{\circ}, 90.0^{\circ}$, and $90.0^{\circ}$, respectively. The tilt angle of R2 is a result of the interplay between the $\pi$ conjugation, which tries to flatten the structure $\left(\varphi=0^{\circ}\right)$, and the steric repulsion of the hydrogen atoms in the ortho positions with respect to the ring-connecting carbons, which favors angles close to $\varphi=90^{\circ} .44$ The methyl groups introduced in S2 and D2 increase the steric repulsion and cause a larger $\varphi$. As a consequence, the conjugation between the phenyl rings is largely broken in S2 and D2, whereas R2 still preserves a reasonable degree of delocalization of the $\pi$-electron system over the whole molecule. This fact is clearly reflected in the change of the gaps $\Delta$ between the highest occupied (HOMO) and lowest unoccupied (LUMO) molecular orbitals, which are $3.85 \mathrm{eV}$ for R2, $4.74 \mathrm{eV}$ for S2, and $4.70 \mathrm{eV}$ for D2. Thus, $\Delta$ increases by roughly $1 \mathrm{eV}$ when 


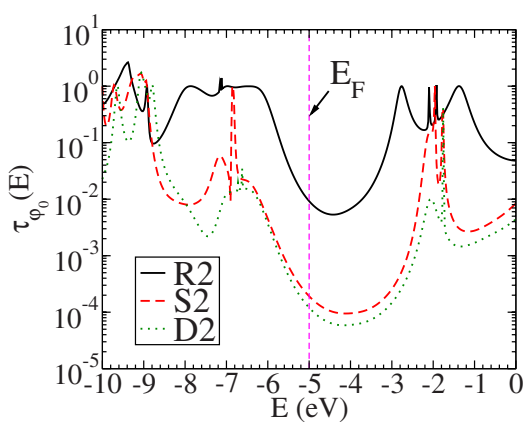

FIG. 4. (Color online) Transmission $\tau_{\varphi_{0}}(E)$ as a function of energy $E$ for the ground-state geometries of the contacts shown in Fig. 2. The low-temperature conductances $G_{\varphi_{0}}(T=0 \mathrm{~K})$ of molecules R2, S2, and D2 are $9.2 \times 10^{-3} G_{0}, 1.9 \times 10^{-4} G_{0}$, and 1.2 $\times 10^{-4} G_{0}$, respectively.

going from $\mathrm{R} 2$ to $\mathrm{S} 2$ or $\mathrm{D} 2$. This suggests that the molecules S2 and D2 will exhibit a more insulating behavior than R2 when they are incorporated into a molecular contact.

\section{B. Low-temperature conductance}

The geometric structures of the metal-molecule-metal contacts are shown in Fig. 2. As there is no stress on the molecule in this binding position, the molecular conformation is very similar to the ground-state structure of the isolated molecule. ${ }^{26}$ In particular, we obtain ground-state tilt angles $\varphi_{0}$ of $33.8^{\circ}, 89.3^{\circ}$, and $89.7^{\circ}$ for R2, S2, and D2, respectively.

In order to analyze the conduction properties of these molecular junctions, we have computed $\tau_{\varphi_{0}}(E)$ for the groundstate geometries of the contacts (Fig. 2). The transmission curves are plotted in Fig. 4, and our result for R2 agrees well with previous theoretical studies. ${ }^{7,41,45}$ Obviously, $\tau_{\varphi_{0}}(E)$ is dominated by a gap around $E_{F}$, which reflects the HOMOLUMO gaps $\Delta$ of the isolated molecules. As can be expected due to the similar geometries of molecules S2 and D2 with $\varphi_{0} \approx 90^{\circ}$, their transmission curves closely resemble each other. However, the most important observation to be made from Fig. 4 is the great reduction of $\tau_{\varphi_{0}}\left(E_{F}\right)$ for S2 and D2 as compared to R2. In particular, for S2 (D2), it is lower than that for R2 by a factor of 48 (77), i.e., roughly by 2 orders of magnitude. This clearly reveals the importance of the conjugated $\pi$-electron system for the charge transport in biphenyl molecules. ${ }^{24}$ In addition, it shows that the conductance can be controlled by means of adequate side groups that force the molecules to adopt different conformations. ${ }^{22}$

Due to their electron-donating nature, ${ }^{46}$ the methyl side groups influence the alignment of the molecular levels with respect to the Fermi energy. In this way slight modifications of the conductance can be achieved. ${ }^{47}$ In Fig. 4, a movement of the HOMO-related peak structure toward more positive energies seems, indeed, visible when going from S2 to D2, where $\varphi_{0}$ remains roughly constant. The absence of a pronounced effect on the conductance can be due to changes in the charge transfer between the molecule and the electrodes ${ }^{42}$ or to the compensation of the shifting effect by a small in- crease in the tilt angle $\varphi_{0}$. Also in Ref. 22, the substituent effect could, apart from the change in $\varphi$, largely be ignored. ${ }^{48}$ It may, however, play a more important role for other observables such as thermopower. ${ }^{49}$

\section{Tilt-angle dependence of ground-state energy and low- temperature conductance}

To investigate the dependence of the conductance on the tilt angle in more detail, we have continuously varied $\varphi$ for all the contacts. We do this by rotating one of the phenyl rings with respect to the other, as illustrated in Fig. 2, without relaxing the contact geometries for $\varphi \neq \varphi_{0}$. We obtain the results depicted in Fig. 5, where the energy $E_{\varphi}$ and the conductance $G_{\varphi}(T=0 \mathrm{~K})$ are plotted as a function of $\varphi \cdot{ }^{50-53} \mathrm{In}$ each case, the angular resolution is $\Delta \varphi=2^{\circ}$.

In the energy curve $E_{\varphi}$ of molecule R2, there are eight extrema visible, four minima and four maxima. They are located at $34^{\circ}, 144^{\circ}, 214^{\circ}$, and $324^{\circ}$ for the energy minima and $90^{\circ}, 178^{\circ}, 270^{\circ}$, and $358^{\circ}$ for the maxima. Due to the symmetry of the molecule, one would expect a $180^{\circ}$ periodicity and a mirror symmetry of both $E_{\varphi}$ and $G_{\varphi}(T=0 \mathrm{~K})$ with respect to $0^{\circ}$ (or, equivalently, $90^{\circ}, 180^{\circ}$, or $270^{\circ}$ ). While the $180^{\circ}$ periodicity is present for $E_{\varphi}$, the mirror symmetry is violated, as one can see in Fig. 5. The reason for this is that the hydrogen atoms have been fixed in their positions with respect to the phenyl rings as obtained for $\varphi=\varphi_{0}$ and, thus, they are standing slightly away from the phenyl ring planes. ${ }^{53,54}$ Contrary to $E_{\varphi}$, all expected symmetries are restored for the conductance. In particular, $G_{\varphi}(T=0 \mathrm{~K})$ possesses only two minima at $90^{\circ}$ and $270^{\circ}$ and two maxima at $0^{\circ}$ and $180^{\circ}$. As a function of the tilt angle, the conductance changes from $2.0 \times 10^{-4} G_{0}$ in the minima to $1.4 \times 10^{-2} G_{0}$ in the maxima; that is, it changes by a factor of 70 . In the case of molecules S2 and D2, $G_{\varphi}(T=0 \mathrm{~K})$ closely follows the shape of $E_{\varphi}$. For S2, there are two minima in $E_{\varphi}$ at $94^{\circ}$ and $268^{\circ}$ with conductances of $2.2 \times 10^{-4} G_{0}$, which are separated by a local maximum at $174^{\circ}$ with a conductance of 1.3 $\times 10^{-2} G_{0}$. This corresponds to a ratio of 60 between the maximum and minimum conductances. D2 exhibits an energy minimum at $90^{\circ}$ and the conductance at this point is $1.2 \times 10^{-4} G_{0}$.

The close agreement of the minima of $G_{\varphi}(T=0 \mathrm{~K})$ for R2, S2, and D2 (Figs. 4 and 5) is remarkable. In the conductance minima, the conformations of these molecules are the same, except for their different side groups and their slightly varying orientations with respect to the gold electrodes. These observations demonstrate again that the side groups mainly control the conformation but have little impact on the low-temperature conductance otherwise. $22,47,49$

The large ratios between maximal and minimal conductances mentioned above highlight the relevance of the extent of the conjugated $\pi$-electron system on the conduction properties of the biphenyl molecules. In order to further investigate this, we have fitted the $G_{\varphi}(T=0 \mathrm{~K})$ curves of Fig. 5 to functions of the form $a+b \cos ^{2} \varphi$ (see the figure caption for the obtained fit parameters). A behavior of $G_{\varphi}(T=0 \mathrm{~K}) / G_{0}$ $\propto \cos ^{2} \varphi$ is expected if the coupling between the $\pi$-electron systems of the two phenyl rings plays the dominant role in 

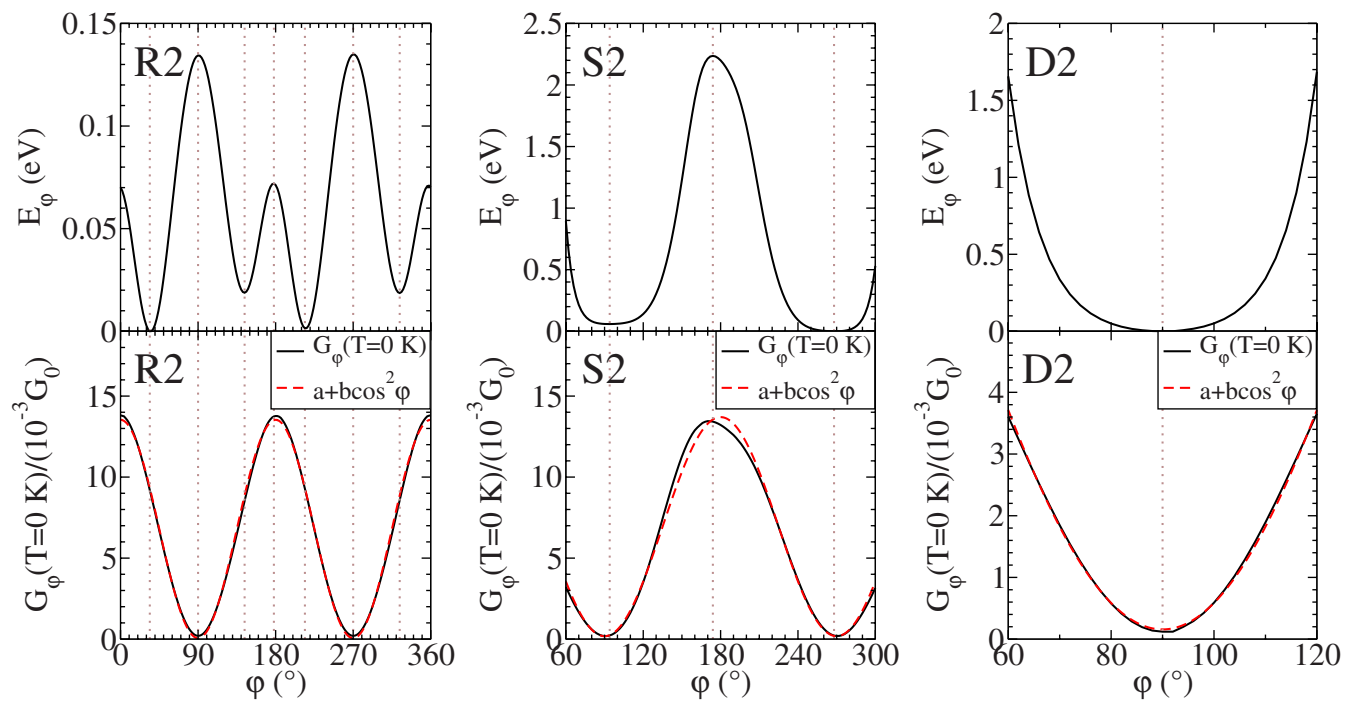

FIG. 5. (Color online) Energy $E_{\varphi}$ (upper panels) and conductance $G_{\varphi}(T=0 \mathrm{~K})$ (lower panels) as a function of tilt angle $\varphi$ for the molecules R2, S2, and D2. Dotted vertical lines indicate positions of extrema in $E_{\varphi}$ for the respective molecules (all panels). In the lower panels, a function of the form $a+b \cos ^{2} \varphi$ has been fitted to $G_{\varphi}(T=0 \mathrm{~K})$ (see the legend). For the fit parameters, we obtain $a=5.95$ $\times 10^{-5} G_{0}$ and $b=1.35 \times 10^{-2} G_{0}(\mathrm{R} 2), a=1.81 \times 10^{-4} G_{0}$ and $b=1.35 \times 10^{-2} G_{0}(\mathrm{~S} 2)$, and $a=1.58 \times 10^{-4} G_{0}$ and $b=1.42 \times 10^{-2} G_{0}(\mathrm{D} 2)$.

charge transport, as discussed in more detail in the Appendix. For all three molecules, our fit matches $G_{\varphi}(T=0 \mathrm{~K})$ very well. What is more, we obtain a very similar parameter $b$ for all of them. On the other hand, the small but positive values of $a$ imply that the conductance at perpendicular tilt angles $\left(\varphi=90^{\circ}\right.$ or $\left.270^{\circ}\right)$ does not vanish entirely, as a pure $\cos ^{2} \varphi$ dependence would suggest. This observation was also made in Ref. 22. The absence of a complete blockade of the transport can be understood by the presence of couplings other than the $\pi-\pi$.

\section{Temperature-dependent conductance and its fluctuations}

Next, we analyze the dependence of the conductance on temperature. In addition to $\bar{G}(T)=\left\langle G_{\varphi}(T)\right\rangle_{\varphi}[\mathrm{Eq}$. (6)], we study $\left\langle G_{\varphi}(T=0 \mathrm{~K})\right\rangle_{\varphi}$. In this way we can quantify the leadinduced contribution to the temperature dependence of $\bar{G}(T)$. To perform the average $\left\langle G_{\varphi}(T=0 \mathrm{~K})\right\rangle_{\varphi}$, we use the energy $E_{\varphi}$ and conductance $G_{\varphi}(T=0 \mathrm{~K})$ of the gold-molecule-gold contacts, as shown in Fig. 5. For $\bar{G}(T)$, instead, we have calculated the transmission function for each angle in an energy interval around $E_{F}$ (see the explanations in Sec. II C) ${ }^{55}$

The temperature-dependent conductances $\bar{G}(T)$ and $\left\langle G_{\varphi}(T=0 \mathrm{~K})\right\rangle_{\varphi}$ are plotted in Fig. 6 for the molecules R2, $\mathrm{S} 2$, and D2 for temperatures $T$ between 0 and $300 \mathrm{~K}$. The average $\bar{G}(T)$ shows qualitative differences for the three molecules considered. S2 and D2 exhibit a rather linear and monotonously increasing conductance $\bar{G}(T)$ with increasing $T$. In contrast, a nonmonotonous weak temperature dependence is found for $\mathrm{R} 2$.

The small differences between $\bar{G}(T)$ and $\left\langle G_{\varphi}(T=0 \mathrm{~K})\right\rangle_{\varphi}$ for S2 and D2 indicate that, for these molecules, the leadinduced contribution to the temperature dependence can be neglected as compared to the configurational one. The mo- notonous increase in $\bar{G}(T)$ can therefore be understood by $E_{\varphi}$ and $G_{\varphi}(T=0 \mathrm{~K})$ (Fig. 5). The ground-state configurations for both molecules correspond to conformations with minimal conductances. Therefore, elevated temperatures give access to conformations with higher conductance values, resulting in the observed steady increase in $\bar{G}(T)$. On an absolute scale, due to the steeper increase in $E_{\varphi}$ for $\varphi \neq \varphi_{0}$, the conductance of D2 changes less than that of S2 when going from 0 to $300 \mathrm{~K}$. This shows that the temperature dependence of the conductance can be suppressed by making a molecule more "rigid." For molecule R2, the situation is qualitatively different. Here, $\varphi_{0}=34^{\circ}$ does not correspond to

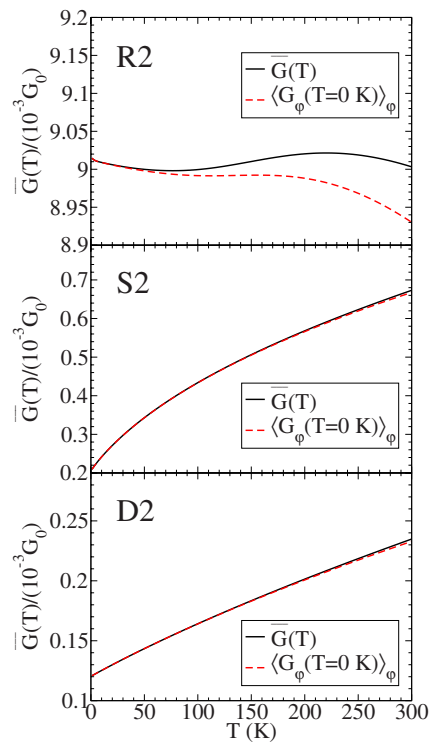

FIG. 6. (Color online) Temperature dependence of the conductances $\bar{G}(T)$ and $\left\langle G_{\varphi}(T=0 \mathrm{~K})\right\rangle_{\varphi}$ for the molecules R2, S2, and D2. 


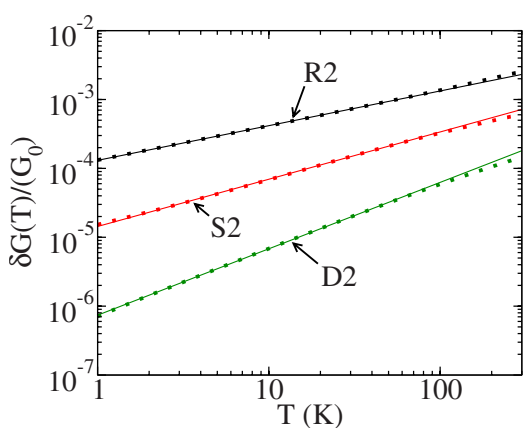

FIG. 7. (Color online) Fluctuations $\delta G(T)$ of the conductance as a function of $T$ for the molecules R2, S2, and D2. Thin lines are best fits to the data with a form $\delta G(T)=\gamma T^{\alpha}$ for the temperature range between 1 and $50 \mathrm{~K}$. We obtain $\gamma=1.31 \times 10^{-4} G_{0}$ and $\alpha$ $=0.50 \quad(\mathrm{R} 2), \quad \gamma=1.43 \times 10^{-5} G_{0}$ and $\alpha=0.69(\mathrm{~S} 2)$, and $\gamma=7.40$ $\times 10^{-7} G_{0}$ and $\alpha=0.96$ (D2).

a minimum of $G_{\varphi}(T=0 \mathrm{~K})$. Elevated temperatures give access to both higher and lower conductances and, as a result, $\left\langle G_{\varphi}(T=0 \mathrm{~K})\right\rangle_{\varphi}$ exhibits no clear trend. The differences between $\bar{G}(T)$ and $\left\langle G_{\varphi}(T=0 \mathrm{~K})\right\rangle_{\varphi}$ at high $T$ signify that for R2, both contributions to the temperature dependence of the conductance, namely, the lead-induced and the configurational ones, play equally important roles. Indeed, they compensate each other and, despite the shallow energy minimum around $\varphi_{0}$, absolute changes in $\bar{G}(T)$ for R2 are even smaller than those for D2.

Finally, we analyze the fluctuations of the conductance $\delta G(T)$ [Eq. (7)], which are plotted in Fig. 7 for temperatures ranging between 1 and $300 \mathrm{~K}$. In each case, $\delta G(T)$ monotonously increases with $T$. This is expected because finite temperatures give access to conductances differing from $G_{\varphi_{0}}(T$ $=0 \mathrm{~K})$ (Fig. 5). It is also evident from Fig. 7 that $\delta G(T)$ is largest for R2 and smallest for D2. Indeed, because of its two methyl side groups, D2 is the most rigid of the three molecules with respect to ring tilts, while R2 can access a large range of conductance values due to its shallow energy landscape (Fig. 6). Since S2 has only one methyl side group, it is an intermediate case. From Fig. 7, we obtain the ratios $\delta G_{\mathrm{R} 2}(T) / \delta G_{\mathrm{S} 2}(T)=4.2$ and $\delta G_{\mathrm{S} 2}(T) / \delta G_{\mathrm{D} 2}(T)=4.2$ for $T$ $=300 \mathrm{~K}$.

\section{E. Harmonic approximation at low temperatures}

To further analyze the differences in the temperature dependence of the three biphenyl molecules, we consider low temperatures and resort to the harmonic approximation $E_{\varphi}$ $=e_{2}\left(\varphi-\varphi_{0}\right)^{2}$. By performing a Sommerfeld expansion of Eq. (5), to second order in $k_{B} T$ we obtain the following:

$$
\bar{G}(T) \approx g_{0}+\frac{g_{2}}{2 e_{2}} k_{B} T+\left(\frac{3 g_{4}}{4 e_{2}^{2}}+\frac{\pi^{2}}{3} G_{0} \tau_{\varphi_{0}}^{(2)}\right)\left(k_{B} T\right)^{2}
$$

and

$$
\delta G(T) \approx\left[\frac{g_{1}^{2}}{2 e_{2}} k_{B} T+\frac{2 g_{2}^{2}+6 g_{1} g_{3}}{4 e_{2}^{2}}\left(k_{B} T\right)^{2}\right]^{1 / 2} .
$$

Here, $g_{n}=\left.\partial_{\varphi}^{n} G_{\varphi}(T=0 \mathrm{~K})\right|_{\varphi=\varphi_{0}} / n$ ! and $\tau_{\varphi_{0}}^{(2)}=\left.\partial_{E}^{2} \tau_{\varphi}(E)\right|_{\varphi=\varphi_{0}} / 2$ !.

Equation (8) demonstrates that at low $T$, the behavior of $\bar{G}(T)$ is determined by two ingredients. The first one is the tilt angle $\varphi_{0}$, which fixes the derivatives $g_{n}$, particularly $g_{2}$. The second ingredient is the curvature $e_{2}$ of $E_{\varphi}$, which measures the rigidity of the biphenyls.

This illustrates the behavior of $\bar{G}(T)$ for S2 and D2 (Fig. $6)$. The ground-state angles $\varphi_{0}$ for these molecules are close to $90^{\circ}$, corresponding to the maximum curvature $g_{2}>0$ in the minimum of the $\cos ^{2} \varphi$-shaped conductance curve (Fig. 5). Consequently, Eq. (8) produces a rather linear increase in $\bar{G}(T)$. As compared to $\mathrm{S} 2$, the higher $e_{2}$ leads to a weaker temperature dependence for D2. The situation is different for R2. Here, $g_{2}$ is negative and smaller than that for S2 and D2 by a factor of around 2. Thus, for low temperature, $\bar{G}(T)$ shows a small linear decrease. At higher $T$, this trend is compensated by the second-order temperature contribution, which increases the conductance $\left(g_{4}, \tau_{\varphi_{0}}^{(2)}>0\right)$. Both effects combine to the constant behavior observed for $\mathrm{R} 2$.

A similar discussion applies for $\delta G(T)$. Since $\varphi_{0} \approx 90^{\circ}$ for D2, the term $g_{1}$ can be ignored. Thus, according to Eq. (9), at low $T$ the fluctuations should follow the form $\delta G(T)=\gamma T^{\alpha}$ with $\alpha \approx 1$. Indeed, from a best fit to the low-temperature region of the curve in Fig. 7, we obtain an exponent $\alpha$ $=0.96$. For R2, the linear term $g_{1}$ does not vanish, however, and we extract an exponent $\alpha=0.50$, which is in agreement with Eq. (9). As $\delta G(T)$ is sensitive to deviations of $\varphi_{0}$ from $90^{\circ}$ and $E_{\varphi}$ is somewhat asymmetric around $\varphi_{0}, \mathrm{~S} 2$ is an intermediate case with $\alpha=0.69$. The prefactors $\gamma$ for the respective molecules can be found in the caption of Fig. 7. Although our fit has been made for low $T$ (between 1 and 50 $\mathrm{K}$ ), deviations from it are small even for elevated temperatures.

\section{DISCUSSION AND CONCLUSIONS}

In experiments with molecular junctions, the conductance on the last plateau of an opening curve is generally attributed to that of a single molecule. In practice, the measured conductances are always time averages over all fast fluctuations of the contact geometry, particularly of the internal configurations of the molecule. The quantity $\bar{G}(T)$ represents such a time average for a given contact realization, while $\delta G(T)$ describes the fast fluctuations. From the curvature of $E_{\varphi}$, we can estimate the vibrational energies $\hbar \omega$ for R2, S2, and D2 to be a few meV. Thus, the time scales of the torsional motion of the molecules are on the order of picoseconds.

The time-averaged conductance may vary considerably from one junction realization to another. ${ }^{2,3,10}$ These variations, and hence the peak widths in conductance histograms, can be attributed to different sources. One is due to changes at the molecule-electrode interface and in the contact environment, while another is due to modifications of the molecule's internal geometric structure. Concerning the first one, 
the surfaces of the metallic electrodes are atomically rough and disordered, and the molecule binds differently to the electrodes in every realization of the junction. ${ }^{8,10,21}$ As a result, the interface-related variability of the conductance is hard to control, although more reproducible results can be achieved by a proper choice of the binding groups. ${ }^{9}$ Regarding the second point, the possibilities of chemical synthesis should be exploited to produce rigid molecules, for example, by means of side groups, as in the examples considered above. ${ }^{17,22}$

In our analysis, several simplifying assumptions have been made. In particular, we have concentrated on a certain realization of a single-molecule junction (Fig. 2) and all temperature-induced changes at the electrode-molecule interface have been neglected. Furthermore, only one configurational degree of freedom of the molecule, the tilt angle $\varphi$, has been considered, and we treated it as a classical variable. At very low temperatures $\left(T \lesssim \hbar \omega / k_{B}\right)$, the quantum-mechanical nature of the torsional vibrations should be taken into account. ${ }^{16}$ Nevertheless, our analysis serves to illustrate the importance of the temperature-related effects on the average conductance and its fluctuations.

In conclusion, we studied charge transport through different dithiolated biphenyl derivatives. By using a densityfunctional-based transport theory, we showed that the conduction through these molecules is strongly influenced by the degree of $\pi$-electron delocalization. A broken conjugation, which is induced by side groups, suppresses the conductance by roughly 2 orders of magnitude. When varying the tilt angle $\varphi$ between the different phenyl rings, we observed a clear $\cos ^{2} \varphi$ dependence of the low-temperature conductance, although the suppression of the conductance for perpendicular ring configurations is not complete. We showed that the methyl side groups in the biphenyl molecules control the conformation, but they have little influence on the lowtemperature conductance otherwise. These findings are in agreement with the experimental results of Ref. 22. In addition, we determined the temperature dependence of the conductance and its fluctuations. Here, we considered two different contributions. The first one originates from the thermal smearing in the leads, while the other one is due to a thermal average over different contact configurations. We observed qualitatively different temperature characteristics for the well-conjugated biphenyl molecule as compared to the molecules whose conjugation was broken by means of methyl side groups. Furthermore, we illustrated that an appropriate design can help reduce temperature-induced conductance fluctuations by stabilizing a molecule in a configuration close to its ground-state structure in isolation. In this way uncertainties with respect to the molecule's internal geometric structure are reduced and a more reliable comparison between theory and experiment appears possible.

\section{ACKNOWLEDGMENTS}

We acknowledge stimulating discussions with M. Mayor, U. Huniar, and D. Rappoport. In addition, we thank R. Ahlrichs for providing us with TURBOMOLE. This work was fi- nancially supported by the Helmholtz Gemeinschaft (Contract No. VH-NG-029), the EU network BIMORE (Grant No. MRTN-CT-2006-035859), the DFG within the CFN, and through the funding of the Young Investigator Group of F.P. at KIT.

\section{APPENDIX: EFFECTIVE $\pi$-ORBITAL COUPLING MODEL}

The dependence of charge transfer on the tilt angle $\varphi$ between two phenyl rings has been previously inspected in Refs. 23 and 24. In this appendix, we explicitly discuss how the $\cos ^{2} \varphi$ behavior of the conductance can be understood based on an effective $\pi$-orbital coupling model within the Green's function formalism.

For this purpose, we bring the transmission function $\tau_{\varphi}(E)$ [Eq. (2)] into a slightly different form, following Ref. 27. We assume that the $C$ part of our contacts can be divided into regions 1 and 2, where region 1 (2) is not coupled to the $R$ $(L)$ part of the system via direct hoppings or overlaps. Furthermore, regions 1 and 2 are connected to each other by $\boldsymbol{t}_{12}=\boldsymbol{H}_{12}-E \boldsymbol{S}_{12}$. Then we may write

$$
\tau_{\varphi}(E)=\operatorname{Tr}\left[\boldsymbol{A}_{11} \boldsymbol{T}_{12} \boldsymbol{A}_{22} \boldsymbol{T}_{21}\right],
$$

where $\quad \boldsymbol{A}_{11}=i\left(\boldsymbol{g}_{11}^{r}-\boldsymbol{g}_{11}^{a}\right) \quad$ and $\quad \boldsymbol{g}_{11}^{r}=\left[\boldsymbol{g}_{11}^{a}\right]^{\dagger}=\left[E \boldsymbol{S}_{11}-\boldsymbol{H}_{11}\right.$ $\left.-\left(\Sigma_{L}^{r}\right)_{11}\right]^{-1}$ are the spectral density and Green's functions of region 1 in the absence of $\boldsymbol{t}_{12}$, and $\boldsymbol{T}_{12}=\boldsymbol{t}_{12}+\boldsymbol{t}_{12}\left(\boldsymbol{G}_{C C}^{r}\right)_{21} \boldsymbol{t}_{12}$. Similar expressions hold for $\boldsymbol{A}_{22}$ and $\boldsymbol{T}_{21}$.

In our case, region 1 (2) is made up of all atoms in the first (second) phenyl ring plus the sulfur and three gold atoms to the left (right) in region $C$ (Fig. 2). To simplify the discussion, we consider the electronic structure of the molecule in the junctions as separable into $\sigma$ and $\pi$ valence electrons, a procedure called $\pi$-electron approximation. ${ }^{56}$ Furthermore, we concentrate on the couplings between those $2 p$ orbitals on the ring-connecting carbon atoms, which contribute to the $\pi$-electron system. These are oriented perpendicular to the respective phenyl rings and are thus rotated by the angle $\varphi$ with respect to each other. The indices 1 and 2 then refer to these $2 p$ orbitals, and the matrices in Eq. (A1) become scalars. Within an extended Hückel model, $H_{12}$ is proportional to the overlap $S_{12}$ (Refs. 57-59) and the scalar coupling element $t_{12}(\varphi)=H_{12}(\varphi)-E S_{12}(\varphi)$ at tilt angle $\varphi$ is seen to be proportional to $\cos \varphi$.

Since the Fermi energy of gold is located in the HOMOLUMO gap of the organic molecules (Fig. 4), $G_{21}^{r}$ can be assumed to be small at $E_{F}$. Therefore $T_{12}(\varphi) \approx t_{12}(\varphi)$. Since the $\varphi$ dependence of $A_{11}\left(A_{22}\right)$ can be expected to be weak, the $\cos ^{2} \varphi$ behavior of the low-temperature conductance follows from Eqs. (5) and (A1),

$$
G_{\varphi}(T=0 \mathrm{~K})=G_{0} \tau_{\varphi}\left(E_{F}\right) \approx\left|t_{12}(\varphi)\right|^{2} A_{11} A_{22},
$$

with all energy-dependent quantities evaluated at $E_{F}$.

Small deviations from the $\cos ^{2} \varphi$ dependence of $G_{\varphi}(T$ $=0 \mathrm{~K})$ can be expected due to higher-order terms in the expansion of $T_{12}$ or couplings other than the $\pi$ - $\pi$ in $\boldsymbol{t}_{12}$. These include, for example, $\sigma-\sigma$ couplings of the ringconnecting carbon atoms and next-nearest-neighbor couplings between regions 1 and 2 . 
*fabian.pauly@kit.edu

${ }^{1}$ N. J. Tao, Nat. Nanotechnol. 1, 173 (2006).

${ }^{2}$ J. Reichert, R. Ochs, D. Beckmann, H. B. Weber, M. Mayor, and H. v. Löhneysen, Phys. Rev. Lett. 88, 176804 (2002).

${ }^{3}$ B. Xu and N. J. Tao, Science 301, 1221 (2003).

${ }^{4}$ Y. Xue and M. A. Ratner, Phys. Rev. B 68, 115407 (2003).

${ }^{5}$ Y. Hu, Y. Zhu, H. Gao, and H. Guo, Phys. Rev. Lett. 95, 156803 (2005).

${ }^{6}$ R. B. Pontes, F. D. Novaes, A. Fazzio, and A. J. R. da Silva, J. Am. Chem. Soc. 128, 8996 (2006).

${ }^{7}$ R. Stadler, J. Phys.: Conf. Ser. 61, 1097 (2007).

${ }^{8}$ G. K. Ramachandran, T. J. Hopson, A. M. Rawlett, L. A. Nagahara, A. Primak, and S. M. Lindsay, Science 300, 1413 (2003).

${ }^{9}$ L. Venkataraman, J. E. Klare, I. W. Tam, C. Nuckolls, M. H. Hybertsen, and M. L. Steigerwald, Nano Lett. 6, 458 (2006).

${ }^{10}$ J. Ulrich, D. Esrail, W. Pontius, L. Venkataraman, D. Millar, and L. H. Doerrer, J. Phys. Chem. B 110, 2462 (2006).

${ }^{11}$ F. Chen, X. Li, J. Hihath, Z. Huang, and N. Tao, J. Am. Chem. Soc. 128, 15874 (2006).

${ }^{12}$ E. Lörtscher, J. W. Cizek, J. Tour, and H Riel, Small 2, 973 (2006)

${ }^{13}$ Z. J. Donhauser, B. A. Mantooth, K. F. Kelley, L. A. Bumm, J. D. Monnell, J. J. Stapelton, D. W. Price, Jr., A. M. Rawlett, D. L. Allara, J. M. Tour, and P. S. Weiss, Science 292, 2303 (2001).

${ }^{14}$ A. Troisi and M. A. Ratner, Nano Lett. 4, 591 (2004).

${ }^{15}$ A. W. Ghosh, T. Rakshit, and S. Datta, Nano Lett. 4, 565 (2004).

${ }^{16}$ M. Čížek, M. Thoss, and W. Domcke, Czech. J. Phys. 55, 189 (2005).

${ }^{17}$ W. Haiss, C. Wang, I. Grace, A. S. Batsanov, D. J. Schiffrin, S. J. Higgins, M. R. Bryce, C. J. Lambert, and R. J. Nichols, Nat. Mater. 5, 995 (2006).

${ }^{18}$ D. R. Jones and A. Troisi, J. Biophys. Biochem. Cytol. 111, 14567 (2007).

${ }^{19}$ X. D. Cui, A. Primak, X. Zarate, J. Tomfohr, O. F. Sankey, A. L. Moore, T. A. Moore, D. Gust, G. Harris, and S. M. Lindsay, Science 294, 571 (2001).

${ }^{20}$ R. H. M. Smit, Y. Noat, C. Untiedt, N. D. Lang, M. C. van Hemert, and J. M. van Ruitenbeek, Nature (London) 419, 906 (2002).

${ }^{21}$ Z. Li, I. Pobelov, B. Han, T. Wandlowski, A. Błaszczyk, and M. Mayor, Nanotechnology 18, 044018 (2007).

${ }^{22}$ L. Venkataraman, J. E. Klare, C. Nuckolls, M. S. Hybertsen, and M. L. Steigerwald, Nature (London) 442, 904 (2006).

${ }^{23}$ S. Woitellier, J. P. Launay, and C. Joachim, Chem. Phys. 131, 481 (1989)

${ }^{24}$ M. P. Samanta, W. Tian, S. Datta, J. I. Henderson, and C. P. Kubiak, Phys. Rev. B 53, R7626 (1996).

${ }^{25}$ A. Shaporenko, M. Elbing, A. Błaszcyk, C. von Hänsch, M. Mayor, and M. Zharnikov, J. Phys. Chem. B 110, 4307 (2006).

${ }^{26}$ F. Pauly, Ph.D. thesis, Institut für Theoretische Festkörperphysik, Universität Karlsruhe, Karlsruhe, 2007; F. Pauly, J. K. Viljas, U. Huniar, M. Häfner, S. Wohlthat, J. C. Cuevas, and G. Schön (unpublished).

${ }^{27}$ S. Wohlthat, F. Pauly, J. K. Viljas, J. C. Cuevas, and G. Schön, Phys. Rev. B 76, 075413 (2007).

${ }^{28}$ J. K. Viljas, F. Pauly, and J. C. Cuevas, Phys. Rev. B 76, 033403 (2007).

${ }^{29}$ R. Ahlrichs, M. Bär, M. Häser, H. Horn, and C. Kölmel, Chem. Phys. Lett. 162, 165 (1989)

${ }^{30}$ K. Eichkorn, O. Treutler, H. Öhm, M. Häser, and R. Ahlrichs,
Chem. Phys. Lett. 242, 652 (1995).

${ }^{31}$ A. Schäfer, H. Horn, and R. Ahlrichs, J. Chem. Phys. 97, 2571 (1992).

${ }^{32}$ K. Eichkorn, F. Weigend, O. Treutler, and R. Ahlrichs, Theor. Chem. Acc. 97, 119 (1997).

${ }^{33}$ A. D. Becke, Phys. Rev. A 38, 3098 (1988).

${ }^{34}$ J. P. Perdew, Phys. Rev. B 33, 8822 (1986).

${ }^{35}$ S. H. Vosko, L. Wilk, and M. Nusair, Can. J. Phys. 58, 1200 (1980).

${ }^{36}$ Y. Q. Xue, S. Datta, and M. A. Ratner, Chem. Phys. 281, 151 (2002).

${ }^{37}$ J. K. Viljas, J. C. Cuevas, F. Pauly, and M. Häfner, Phys. Rev. B 72, 245415 (2005).

${ }^{38}$ S. Datta, Electronic Transport in Mesoscopic Systems (Cambridge University Press, Cambridge, 1995).

${ }^{39}$ F. Guinea, C. Tejedor, F. Flores, and E. Louis, Phys. Rev. B 28, 4397 (1983).

${ }^{40}$ Y. Xue, S. Datta, and M. A. Ratner, J. Chem. Phys. 115, 4292 (2001).

${ }^{41}$ Y. Xue and M. A. Ratner, Phys. Rev. B 68, 115406 (2003).

${ }^{42}$ R. Stadler and K. W. Jacobsen, Phys. Rev. B 74, 161405(R) (2006).

${ }^{43}$ We neglect temperature-dependent changes of the chemical potential since they are negligibly small (Ref. 60).

${ }^{44}$ L. F. Pacios and L. Gómez, Chem. Phys. Lett. 432, 414 (2006).

${ }^{45}$ M. Kondo, T. Tada, and K. Yoshizawa, J. Phys. Chem. A 108, 9143 (2004).

${ }^{46}$ J. Clayden, N. Greeves, S. Warren, and P. Wothers, Organic Chemistry (Oxford University Press, Oxford, 2001).

${ }^{47}$ L. Venkataraman, Y. S. Park, A. C. Whalley, C. Nuckolls, M. S. Hybertsen, and M. L. Steigerwald, Nano Lett. 7, 502 (2007).

${ }^{48}$ C. M. Finch, S. Sirichantaropass, S. W. Bailey, I. M. Grace, V. M. García-Suárez, and C. J. Lambert, J. Phys.: Condens. Matter 20, 022203 (2008).

${ }^{49}$ F. Pauly, J. K. Viljas, and J. C. Cuevas, arXiv:0709.3588 (unpublished).

${ }^{50}$ The smaller tilt-angle intervals for $\mathrm{S} 2$ and D2 as compared to that for R2 are chosen to prevent the methyl groups from coming too close to each other. For S2, we rotate the molecule such that each methyl group passes a hydrogen atom, but we avoid tilt angles where the two methyl groups would meet. Accordingly, for $\mathrm{D} 2, \varphi$ is restricted even further.

${ }^{51}$ Fixing all degrees of freedom, except for the tilt angle $\varphi$, most likely leads to an overestimation of rotational energy barriers (Refs. 52 and 53).

${ }^{52}$ A. Almenningen, O. Bastiansen, L. Fernholt, B. N. Cyvin, S. J. Cyvin, and S. Samdal, J. Mol. Struct. 128, 59 (1985).

${ }^{53}$ S. Arulmozhiraja and T. Fujii, J. Chem. Phys. 115, 10589 (2001).

${ }^{54}$ J. C. Sancho-García and J. Cornil, J. Chem. Phys. 121, 3096 (2004).

${ }^{55} \mathrm{We}$ take the thermal average $\bar{G}(T)=\left\langle G_{\varphi}(T)\right\rangle_{\varphi}$ only over symmetry-nonredundant tilt-angle intervals, in which $\varphi_{0}$ of the contacts of Fig. 2 is contained. These intervals are $\left[0^{\circ}, 90^{\circ}\right]$, $\left[180^{\circ}, 300^{\circ}\right]$, and $\left[60^{\circ}, 120^{\circ}\right]$ for $\mathrm{R} 2, \mathrm{~S} 2$, and D2, respectively (Fig. 5). The averages in Eqs. (6) and (7) are performed as discrete sums. We used angle resolutions down to $\Delta \varphi=0.1^{\circ}$ to resolve well the energy minima of $E_{\varphi}$. 
${ }^{56}$ D. M. Bishop, Group Theory and Chemistry (Dover, New York, 1993).

${ }^{57}$ M. Wolfsberg and L. Helmholz, J. Chem. Phys. 20, 837 (1952).

${ }^{58}$ R. Hoffmann, J. Chem. Phys. 39, 1397 (1963).
${ }^{59}$ J. Haasea, R. Janoschek, H. Preussc, and G. Diercksen, J. Mol. Struct. 3, 165 (1969).

${ }^{60}$ N. W. Ashcroft and N. D. Mermin, Solid State Physics (Harcourt College, Orlando, 1976). 